\title{
Disaggregated optical network control and orchestration of heterogeneous domains
}

\author{
P. Castoldi*, S. Fichera*, M. Gharbaoui ${ }^{\S}$, A. Giorgetti*, B. Martini ${ }^{\S}$, F. Paolucci* \\ *Scuola Superiore Sant'Anna, Pisa, Italy; ${ }^{\$}$ CNIT, Pisa, Italy \\ [p.castoldi, s.fichera, a.giorgetti, fr.paolucci]@santannapisa.it; [molka.gharbaoui, barbara.martini]@cnit.it
}

\begin{abstract}
Network softwarization and disaggregation are two trends that are revolutionizing the network-cloud ecosystem. This paper details possible solutions to control and monitor an infrastructure including an IoT domain, a Cloud domain and a packet-optical network domain.

Keywords: Disaggregation, NFV/SDN techniques, optical corelaccess networks.
\end{abstract}

\section{INTRODUCTION}

The increasing availability of miniaturized sensors with computing, storage and networking capabilities, e.g., Internet of Things (IoT), and the pervasive diffusion of 5G, portend a scenario with a rich set of interconnected smart terminals (e.g., robots, sensors, smart cars) working together to enable novel and enriched IoT services (e.g. smart cities, industrial automation). Contextually, SDN and NFV emerged as the breaking technologies for the telecommunication industry since they bring the use of standard hardware, cloud computing technologies (i.e., software virtualization) and on-demand provisioning mechanisms into Telco deployments (e.g., Cloud RAN). This systemic transformation of telecommunications and ICT, called softwarization, will make the major impact at the Edge of current telecommunication networks resulting in a convergent network-cloud ecosystem [1].

The softwarization process is also involving the optical layer of communication networks (both in metro and core segments) where the SDN concepts are gradually being deployed [8,9] together with the feature of data plane disaggregation [10] that allows separate control of the many components of the optical layer (e.g., transponders and optical nodes). This disaggregation process is strongly pushed by telecommunication providers because it breaks the vendor lockin dependencies and allows flexible and gradual update/upgrade of the network. As a typical example, the technological evolution of transponders (e.g., increase of supported bitrates) is much faster with respect to the evolution of optical nodes (e.g., ROADMs). Thus, in a disaggregated network, transponders can be replaced more frequently and considering all the products on the market (i.e., no vendor lock-in) to keep the network efficiency aligned with the technological frontier.

As shown in Fig.1, the resulting scenario is a dense deployment of micro-clouds spread out at the Edge, composed of generalized Virtual Functions (VFs) providing both network and application services according to the "as-a-service" paradigm where VFs can be exposed as service components with APIs and dynamically selected, composed and provisioned (i.e., orchestrated). The composite set of VFs (i.e., service chain) is executed in a "slice" which is made of a set of logical resources (i.e., VMs or Containers) interconnected by a set of Virtual Links [1]. In turn, the interconnection network includes two main technologies: packet-based devices (e.g., SDN switches) and optical devices (e.g., ROADMs and optical transponders). In such a scenario, the orchestration process is required to address the application demands by coordinately provision a composite set of services across different administrative and/or technological domains (e.g., cloud and network domains, different service provider domains, packet-based and optical network domains), while automating the tasks of composing and exposing them as a single service instance [2].

The orchestration is expected to guarantee the adequate service performance according to the expected QoS during the service lifecycle despite concurrent resource usage among users or possible service outages. To this purpose, in addition to the provisioning functions, the orchestration process also relies on monitoring and profiling functions to measure the status of the underlying (both physical and virtual) resources (e.g., amount traffic processed by packet-based devices, quality of the physical signal on the optical layer). Indeed, based on feedback from monitoring tools, the orchestration is expected to handle exceptions or deviations from normal workflows and to adapt provisioned resources to recover from service degradations or outages [3].

This paper focuses on the SDN control and monitoring of optical disaggregated networks and on the orchestration at the network level across IoT, Cloud and Edge network domains while exploiting programmability features offered by SDN to dynamically adapt service chain paths upon either congestion events at switches/links or SLA violations of flows to assure the proper level of data delivery to VFs.

\section{SDN CONTROL AND MONITORING OF DISAGGREGATED OPTICAL NETWORKS}

The utilization of SDN on optical networks is still in a development phase and in course of experimentation in research environments [10]. The first attempt to enable SDN to control an optical network considered the extension of the OpenFlow protocol [8]. However, OpenFlow is not flexible enough to encompass the whole variety of configurations required on the optical layer (e.g., modulation formats supported by the transponders. frequency grids supported by optical 


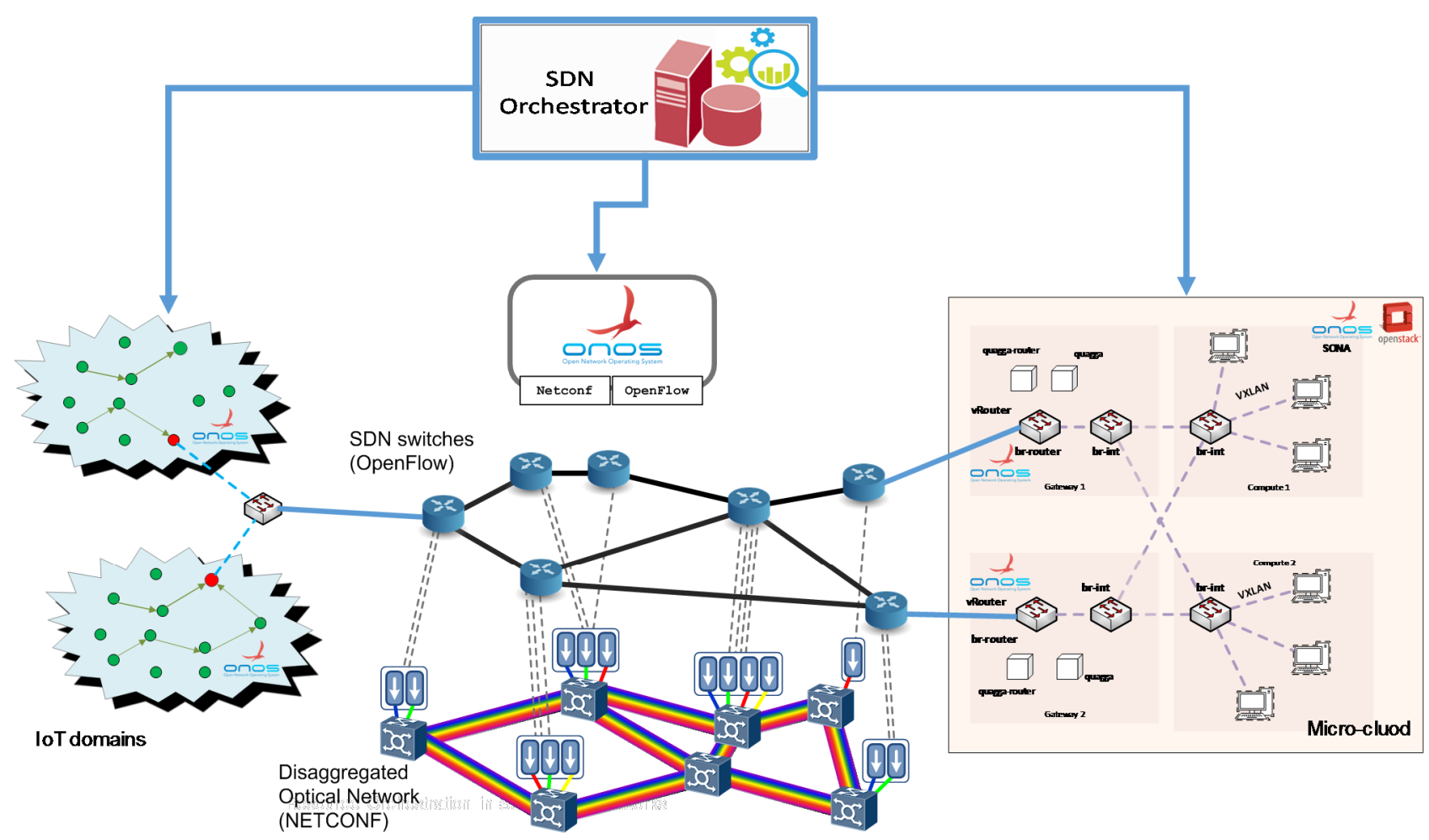

Fig. 1: Testbed set-up with SDN-enabled IoT; a two layer transport network including a packet switched domain (SDN switches) and a disaggregated optical network domain (i.e., composed of optical transponders and ROADMs); and a Cloud domain.

filters). In the meanwhile, YANG modeling has been adopted in several standardization consortia (e.g., OpenConfig, OpenROADM) for the complete characterization of optical devices and the related configuration procedures. With this kind of modeling language, the natural protocol to control and monitor the network became the NETCONF protocol [8]. For instance, the ODTN working group has been created within ONF to enable the ONOS SDN framework [7] to control, configure and monitor disaggregated optical networks based on NETCONF/YANG. Specifically, the ODTN work is planned in three main phases considering increasing levels of data-plane disaggregation. In a first phase the optical network is considered to be disaggregated in transponders and an OLS (i.e., Open Line System), then the support of ROADMs will be introduced so that a meshed network topology can be correctly configured by the controller; finally, the ROADMs will be further disaggregated in more elementary devices such as, node degrees, filters, optical amplifiers, that could be individually configured and monitored by the SDN controller.

The implementation of effective monitoring mechanisms and their integration with the controller is therefore of fundamental importance, so that the network can be dynamically re-configured by the controller in presence of specific events that may occur on the data plane. For instance, monitoring of the OSNR should be employed so that soft-failures on the optical fibers can be detected on time and traffic can be deviated on alternate routes [10]. However, those monitoring functionalities cannot be directly integrated within the controller because they risk to overload it dramatically compromising the other controller functionalities.

Fig. 2 reports our proposal for the integration of the ONOS controller with external monitoring tools. Specifically, the controller activates connectivity using NETCONF protocol (1-2-3); an alarm is notified from a receiver to the controller with NETCONF notifications, i.e., OSNR degradation on a specific lightpath (4); the controller activates detailed gRPC monitorig on some points of the network, i.e., on the source and destination transponders of the lightpath (6); after data analysis the monitoring tools provide a feedback to the controller, i.e.,re-route the lighpath on an alternate route (7).

\section{SDN ORCHESTRATION ACROSS EDGE NETWORK, CLOUd AND IOT DOMAINS}

The presented SDN orchestrator provisions service chain paths connecting VFs deployed across IoT, the Cloud and Edge network domains, while adapting them in order to assure both reliable and throughput-assured data delivery during the service lifecycle. The SDN orchestrator performs an orchestration process at the network infrastructure level that allows for the programmable provision of service chain paths connecting VFs, internally represented/handled as endpoint IP addresses. The SDN orchestrator has been implemented as an application running on top of ONOS controllers in charge of offering control capabilities of the underlying network nodes through API. The SDN orchestrator exposes a RESTful interface which allows for asking the set-up and the teardown of service chain paths connecting specified endpoints (also traversing a set of VF end-points, if specified) by using intent-based semantics without the need to specify technology-specific low-level details. Then, the underlying controllers handle the low-level directives (i.e., OF messages) 
to switches. Moreover, the SDN orchestrator collects traffic statistics from the switches and elaborates them to evaluate their current load. In case of congestion events or SLA violations (i.e., QoS degradations), it activates recovery mechanisms through path adaptations, i.e., redirection of (part of) service chain paths. Further details on the SDN orchestrator can be found in [4].

The proposed orchestration solutions have been evaluated on a laboratory testbed that reproduces an SDN infrastructure across IoT, Cloud and Edge Network domains. The IoT domain is composed of a number of networked smart devices (i.e., sensors) that can be deployed in different environments (e.g., roads, medical centers, and farms) to collect data (e.g., temperature data). The SDN Edge Network connects a number of Fog systems and serves as an interconnecting/transit domain between the IoT and the Cloud resource domain (i.e., OpenStack). We consider that the traffic is generated from the IoT devices and sent to the gateway to traverse the SDN domain and then to reach the VFs deployed as VMs in the OpenStack. Both IoT and Cloud domain deploy an SDN network to connect sensors and VMs, respectively, using WISE and SONA frameworks [5][6], while we emulate the SDN transit network domain using Mininet where we specified the set of OpenFlow switches and the links capacities. We sent a set of path set-up requests generated randomly according to a Poisson process. The source end-host of every request represents a node from the IoT domain while the destination end host is a VM in the OpenStack domain. Once the data delivery path is established, we start sending traffic from the source to the destination for the whole duration of the service time.

In Fig. 3 and Fig. 4, we show the evolution in time of the average load in all the monitored switches. We can notice that while with redirection the throughput increases in time in an almost uniform way for all the switches, when no redirection is performed the load increase is very high in some switches while it is low or even null in others.

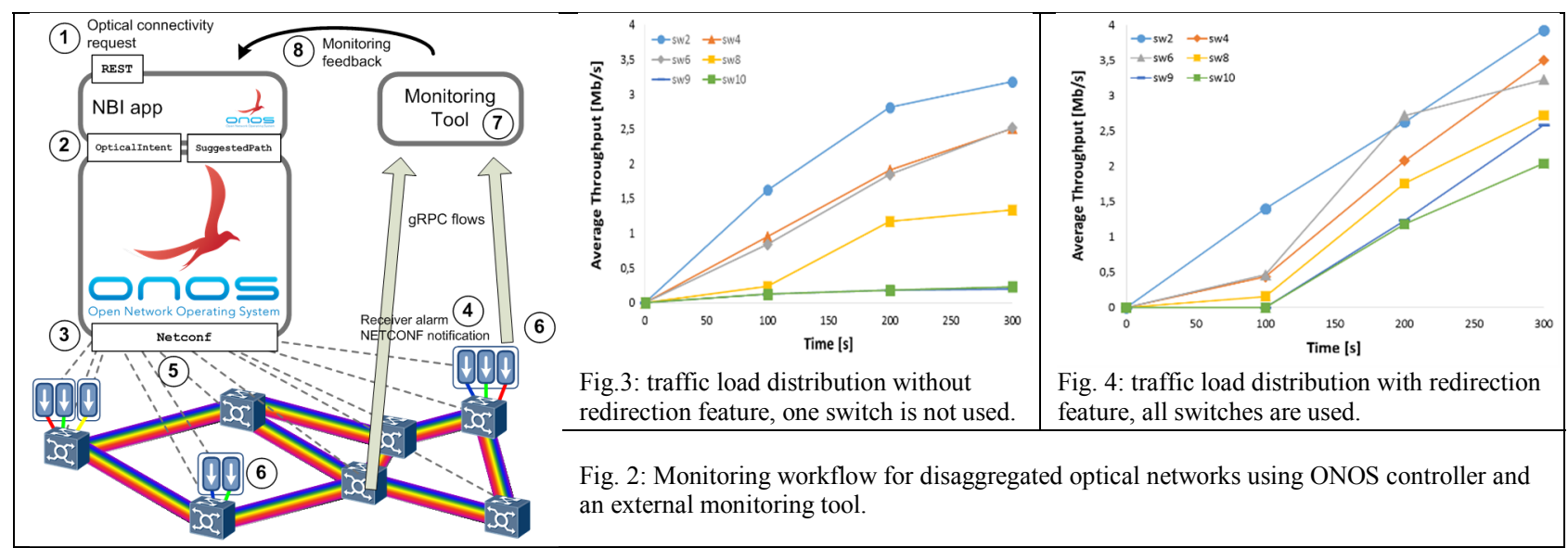

\section{CONCLUSION}

This work focused on the SDN control and monitoring of optical disaggregated networks and on the orchestration at the network level across IoT, Cloud and Edge network domains while exploiting programmability features offered by SDN. Specifically, an architecture for the integration of external monitoring tools within ONOS has been proposed to be applied in a disaggregated optical network scenario. Moreover, experimental results of traffic load balancing triggered by a monitored event is demonstrated on a testbed composed of emulated SDN devices.

\section{REFERENCES}

[1] A. Manzalini et al, "An edge operating system enabling anything-as -a-service," in IEEE Comm. Mag., Mar.2016.

[2] D. Dietrich, et al., "Multi-Provider Service Chain Embedding With Nestor," in IEEE Transactions on Network and Service Management, vol. 14, no. 1, pp. 91-105, March 2017.

[3] B. Martini, et al., "Cross-Functional resource orchestration in optical telco clouds," 2015 ICTON, Budapest, Hungary, pp. 1-5.

[4] M. Gharbaoui et al. "Network Orchestrator for QoS-enabled Service Function Chaining in reliable NFV/SDN infrastructure," IEEE NetSoft, Bologna, Italy 2017.

[5] http://sdn-wise.dieei.unict.it/

[6] https://wiki.onosproject.org/display/ONOS/SONA+Architecture

[7] A. Giorgetti, F. Paolucci, F. Cugini, P. Castoldi, "Dynamic restoration with GMPLS and SDN control plane in elastic optical networks", in IEEE/OSA Journal of Optical Communications and Networking, vol. 7, n. 2, Feb. 2015.

[8] A. Giorgetti, et. al. "ONOS-controlled Disaggregated Optical Networks", in Proc. OFC, March 2019.

[9] R. Casellas, R. Martinez, R. Vilalta, R. Munoz, "Metro-haul: SDN control and orchestration of disaggregated optical networks with model-driven development", in Proc. ICTON, Jul. 2018.

[10] F. Paolucci, A. Sgambelluri, F. Cugini, P. Castoldi, "Network telemetry streaming services in SDN-based disaggregated optical networks", IEEE/OSA Journal of Lightwave Technology, vol. 36, n. 15, Aug. 2018. 